\title{
Methods for Tractography-Driven Surface Registration of Brain Structures
}

\author{
Aleksandar Petrović, Stephen M. Smith, Ricarda A. Menke, \\ and Mark Jenkinson \\ Centre for Functional MRI of the Brain (FMRIB), University of Oxford \\ petrovic@fmrib.ox.ac.uk
}

\begin{abstract}
Registration of brain structures should bring anatomically equivalent areas into correspondence which is usually done using information from structural MRI modalities. Correspondence can be improved by using other image modalities that provide complementary data. In this paper we propose and evaluate two novel surface registration algorithms which improve within-surface correspondence in brain structures. Both approaches use a white-matter tract similarity function (derived from probabilistic tractography) to match areas of similar connectivity patterns. The two methods differ in the way the deformation field is calculated and in how the multi-scale registration framework is implemented. We validated both algorithms using artificial and real image examples, in both cases showing high registration consistency and the ability to find differences in thalamic sub-structures between Alzheimer's disease and control subjects. The results suggest differences in thalamic connectivity predominantly in the medial dorsal parts of the left thalamus.
\end{abstract}

\section{Introduction}

Different MRI modalities provide complementary data, informing us in various ways about brain anatomy and physiology. It is therefore natural to try to interpret them in an integrated fashion. This is beneficial for a variety of reasons; for example, matching structural landmarks (especially in the cortex) does not always imply matching functional regions [1]. Also, some structures appear featureless in a certain modality, with no particular structural landmarks for matching, whereas other modalities may provide clearer information (e.g. white matter changes in DTI data).

We believe that image registration can be improved if, in addition to matching T1-weighted image intensities, we also try to align brain regions according to their connectivity patterns (information from diffusion-weighted images). Most importantly, including connectivity information takes us closer to matching functional areas 234 . In this case, the description of every brain region becomes more specific, which should result in better matching accuracy across the population.

In this paper we propose two registration methods that integrate information available in T1-weighted images and probabilistic tractography. They use geometrical models of subcortical brain structures (e.g., human thalamus is used 
here) segmented using the FIRST/FSL software tool [5] to enhance anatomical correspondence between their surfaces. Anatomical alignment is based on intersubject matching of areas of similar connectivity, which supposes that anatomically equivalent areas are connected to the same cortical and sub-cortical regions in different subjects. This is different from [4] where the cortical surface is deformed based on regional connectivity measures to cortical ROIs. We illustrate the effectiveness of our registration methods by applying them to an Alzheimer's disease (AD) data-set, specifically looking for thalamic connectivity alterations, which could have value in determining MRI biomarkers of disease progression.

\section{Methods}

The registration process tries to improve within-surface correspondence, which means that the shape of the extracted structures (represented by meshes output by FIRST) is unchanged by the surface matching. What changes is the correspondence between vertices of the two meshes, which represents anatomical correspondence between thalamic surfaces. For example, let every vertex in the thalamic mesh have a unique label (i.e., a number between 1 and $N$ ) and denote the set of vertices on the surface of the anterior dorsal thalamic nucleus as $S_{1}$ in one subject and $S_{2}$ in the other (in general, different sets of numbers). The purpose of the algorithm is to match vertices in $S_{1}$ to those in $S_{2}$ by sliding them around the surface and in that way re-establish correspondence of labels.

Both registration methods presented here use the same form for the similarity function which measures similarity in white-matter (WM) connectivity of certain brain regions to the rest of the brain. In our case, these brain regions are represented by different points of the mesh delineating the sub-cortical structure. Therefore, the first step in the processing pipeline is to segment matching structures (using FIRST) from T1-weighted images of both subjects (or more if group registration is performed). Meshes delineating segmented structures have a fixed number of vertices (e.g., the thalamus has 642 vertices) that, in our approach, become seeding points for whole-brain probabilistic tractography [6].

Next, we label probabilistic tractography output from vertex $A$ as the "Connectivity Profile" of $A$ : $\mathbf{C P}(A)$. Therefore, $\mathbf{C P}(A)$ is an image where every voxel encodes the number of white-matter tracts originating at coordinates of vertex $A$ and passing through that voxel. The maximum value is the number of random tractography samples at the seed, which is 5000. In the next step, CPs of all vertices for both subjects' structures (human thalami) are transformed into standard MNI space where they can be compared.

After these steps the Correlation Matrix (CM) is formed, which encodes the similarity measure between CPs (reshaped into vectors). We define correlation of vectors $\mathbf{X}$ and $\mathbf{Y}$ as

$$
\operatorname{Corr}(\mathbf{X}, \mathbf{Y})=\frac{\mathbf{X}^{T} \mathbf{Y}}{\left(\mathbf{X}^{T} \mathbf{X}+\mathbf{Y}^{T} \mathbf{Y}\right) / 2}
$$

so that the Correlation Matrix can be written as 


$$
C M(i, j)=\operatorname{Corr}\left(\mathbf{C P}\left(\mathbf{x}_{i}\right), \mathbf{C P}\left(\mathbf{y}_{j}\right)\right), i, j \in[1, N]
$$

where $\mathbf{x}_{i}$ is the $i$ th vertex of a mesh delineating the thalamus in subject $X\left(\mathbf{y}_{j}\right.$ for subject $Y$ ) and $N$ is the number of vertices in each mesh. Values from the $\mathrm{CM}$ are used to derive the similarity function that drives the registration.

FIRST sub-cortical meshes are initially formed by deforming a spherical mesh. We use this equivalence in mesh topology to perform registration using spherical meshes. Registration starts by generating two identical regular spherical meshes (generated by subdividing an icosahedron) representing the input and the reference structure. The reference mesh (sphere) remains undeformed during registration whereas the input mesh undergoes tangential deformations to maximise the similarity function and better match the reference mesh. In this way, vertices from the input mesh move along the surface until they converge to a position where correlations between their CPs and the CPs of the corresponding vertices of the reference mesh are maximised. In the end, the deformation (of the input mesh) is propagated back from the sphere to the mesh of the structure in the native image space.

\subsection{Registration Using Deformation Forces (Method1)}

In this approach we make use of two deformation forces: the similarity force $(\mathrm{SF})$ and the regularisation force $(\mathrm{RF})$. The similarity force aims to maximise similarities between connectivity profiles, whereas the regularisation force aims to preserve the separation of the vertices (Fig. 1). A multi-scale registration framework is realised by grouping vertices into "patches". A "patch" represents a group of vertices that move together. Large patches are crucial at the initial stages of the registration when dealing with large-scale deformations e.g., global rotation. On the other hand, small patches (one vertex is the smallest) only act locally, affecting local mesh features. In total, optimisation is performed at 10 different scales (patch sizes).

The local similarity function (LSF) calculates the mean correlations between all vertices within a patch in the input mesh and the corresponding vertices of the

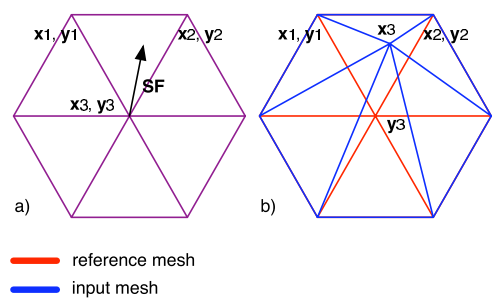

Fig. 1. The effect of the similarity force on an input mesh vertex $\left(\mathbf{x}_{i}-\right.$ input mesh; $\mathbf{y}_{i}$ - reference mesh). The deformation of $\mathbf{x}_{3}$ due to the similarity force is shown. In a) reference and input meshes initially overlap. In b) the similarity force SF moves $\mathbf{x}_{3}$ away from $\mathbf{y}_{3}$ and towards $\mathbf{y}_{2}$ as $\operatorname{Corr}\left(\mathbf{C P}\left(\mathbf{x}_{3}\right), \mathbf{C P}\left(\mathbf{y}_{2}\right)\right)>\operatorname{Corr}\left(\mathbf{C P}\left(\mathbf{x}_{3}\right), \mathbf{C P}\left(\mathbf{y}_{3}\right)\right)$. The regularisation forces would have the opposite effect, bringing $\mathbf{x}_{3}$ closer to $\mathbf{y}_{3}$. 
reference mesh. The similarity force, $\mathbf{S F}$, is defined as the spatial derivative of the LSF with respect to the parameters encoding the position of the patch centre in the input mesh. This force acts equally on all the vertices within a patch, inducing coherent movement. The regularisation force is defined as $\mathbf{R F}\left(\mathbf{x}_{i}, \nu_{i}\right) \propto \mathbf{m}_{i}-\mathbf{x}_{i}$ where $\nu_{i}$ is the set of first neighbours of $\mathbf{x}_{i}$ in $X$ and $\mathbf{m}_{i}$ is the average position of the neighbours, given by $\mathbf{m}_{i}=\frac{1}{\left|\nu_{i}\right|} \sum_{k \in \nu_{i}} \mathbf{x}_{k}$ as in [7]. RF is calculated and applied separately to each vertex within a patch.

$\mathrm{RF}$ and SF are iteratively applied to patches until convergence. The patch sizes (number of vertices) we use are 642, 321, 100, 50, 40 30, 20, 10, 5, 3 and 1. At the smallest patch size, both forces act on one vertex in turn. During every iteration, each vertex becomes the centre of a patch once and is chosen at random. The number of iterations has been empirically optimised using artificial testing data as described below.

Displacements are determined from the forces by scaling parameters, $\lambda$ and $\mu$ for RF and SF respectively, where the values are determined empirically. When a force is applied to a vertex, mesh-intersections and local topology breaches are prevented by checking for intersections and, when they occur, reducing the size of the movement until no intersections occur.

\subsection{Registration Based on Spherical Wavelets (Method2)}

In this registration method we represent the deformation field using a set of biorthogonal wavelet basis functions defined on a sphere [89]. The basis set is constructed of scaling functions at the coarsest scale and wavelet functions at finer scales. Both scaling $(\varphi)$ and wavelet functions $(\psi)$ are defined as $\varphi_{j, k}, \psi_{j, k}$ : $\mathbb{S} \rightarrow \mathbb{R}$, where $\mathbb{S}$ is a unit sphere, $j$ defines the scale of the function and $k$ refers to the spatial index which describes where on the surface the function is centered. At a particular scale $j$, wavelet functions are combinations of scaling functions at scales $j$ and $j+1$. A given function $f: \mathbb{S} \rightarrow \mathbb{R}$ can be expressed as a linear combination of the basis functions

$$
f(\mathbf{x})=\sum_{k} \lambda_{0, k} \varphi_{0, k}(\mathbf{x})+\sum_{j \geq 0} \sum_{m} \gamma_{j, m} \psi_{j, m}(\mathbf{x}) .
$$

Scaling coefficients $\lambda_{0, k}$ represent the low-pass content of the signal $f$ whereas coefficients $\gamma_{j, m}$ represent the localised band-pass content of the signal.

The idea of multi-scale registration using spherical wavelets relies on approximating the function $f$ at different scales - coarse (low) scale encodes large deformations while higher scales determine fine features of the deformation field (for applications in shape analysis see, e.g., [1011]). If a spherical mesh has $N$ vertices, a total of $N$ basis functions are created, composed of $N_{0}$ scaling functions (where $N_{0}$ is the initial number of vertices of the base mesh - e.g. icosahedron) and $N_{r}$ wavelet functions. If each of these basis functions is evaluated in each of the $N$ vertices and these data stacked into a matrix $\Phi_{N \times N}$, every finite energy scalar function $F$ evaluated at $N$ vertices can be transformed into a vector of basis coefficients $\Gamma$ using Forward Wavelet Transform $\Gamma=\Phi^{-1} F$ and recovered using Inverse Wavelet Transform: $F=\Phi \Gamma$. 
If $F$ encodes displacements in Euler angles for every vertex of the input mesh, coefficients in $\Gamma$ can be numerically solved for by maximising the similarity function between CPs of the input $(X)$ and reference $(Y)$ meshes. The similarity function (SFun) is calculated for all vertices of the reference mesh and can be written as

$$
\operatorname{SFun}(X, Y)=\mu \frac{1}{N} \sum_{l \in Y} \widetilde{C M}\left(\mathbf{x}_{l^{*}}, \mathbf{y}_{l}\right)+\nu E_{m}(F),
$$

where $\widetilde{C M}\left(\mathbf{x}_{l^{*}}, \mathbf{y}_{l}\right)$ is the interpolated value of the CP correlation between $\mathbf{y}_{l}$ and the corresponding input mesh vertex $\mathbf{x}_{l^{*}}$, where this position directly depends on the deformation field, $F$. The interpolation of $\mathrm{CM}$ values is achieved using linear interpolation. $E_{m}$ is the membrane energy of the deformation field, taking on the role of the regulariser, while $\mu$ and $\nu$ are scaling parameters found empirically. In order to penalise local topology breaches, parameter $\nu$ is increased (by 10\%) when the deformation field $F$ results in a mesh-intersection.

We use variable metric non-linear optimisation to estimate coefficients $\lambda$ and $\psi(\Gamma)$ 12. This is performed in four steps (on four scales) with all coefficients set to 0 initially. We denote a set of coefficients at scale $i$ by $S_{i}, i \in[0,3]$ (where $\left.S_{i-1} \subset S_{i}\right)$. The first stage of optimisation finds the coefficients at the coarsest scale, $S_{0}$, followed by stages for increasingly higher scales: $S_{1}, S_{2}$ and $S_{3}$.

\section{Results}

In order to test the algorithms and tune free parameters we ran experiments on a set of artificial examples. The artificial meshes are regular spheres with six differently coloured regions (Fig. 2). All vertices of the same colour have identical CPs across spheres/subjects, while vertices with different colours have completely uncorrelated CPs. In order to test the quality of registration we used all spheres from Fig. 2 and performed $\mathrm{A}$ to $\mathrm{B}, \mathrm{B}$ to $\mathrm{C}$ and $\mathrm{C}$ to $\mathrm{A}$ registrations. If we denote the corresponding mappings by $w_{A}, w_{B}$ and $w_{C}: A \stackrel{w_{A}}{\longrightarrow} B \stackrel{w_{B}}{\longrightarrow} C \stackrel{w_{C}}{\longrightarrow} A$, then in an ideal case, $w_{C} \circ w_{B} \circ w_{A}=I$ where $I$ is an identity mapping (we shall call this an $A B C A$ test). Therefore, the residual deformation field of the $A B C A$ test is a good measure of the registration consistency although it cannot be used to assess absolute accuracy. We performed the $A B C A$ test using Methods 1 and 2
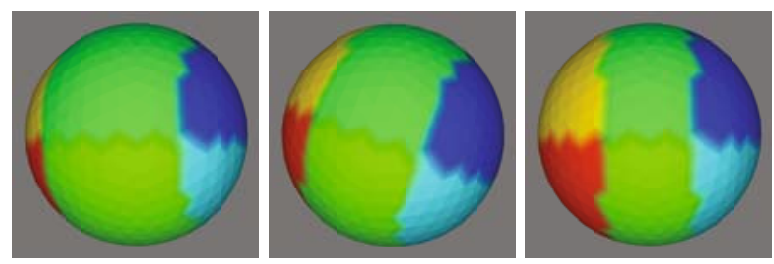

Fig. 2. A (left), B (middle) and C (right) represent three different artificial subjects/spheres. Sizes of the coloured regions and overall rotation vary between subjects. 


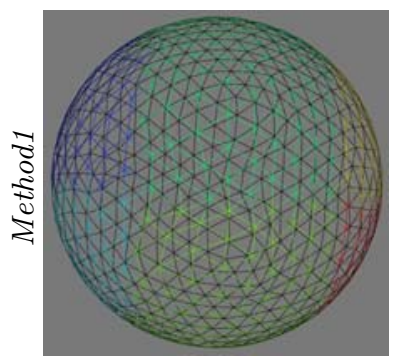

(a)

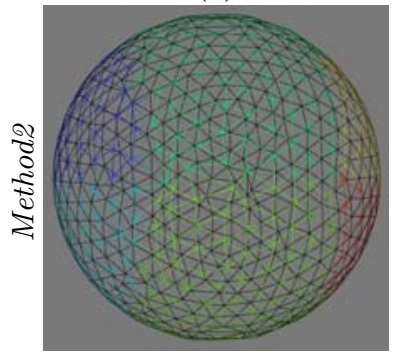

(d)

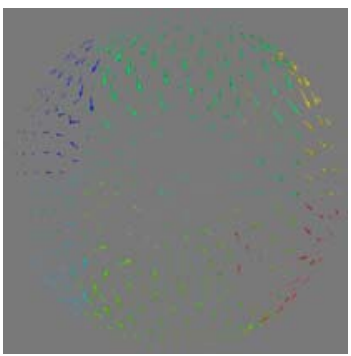

(b)

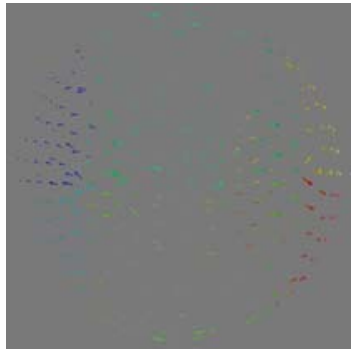

(e)

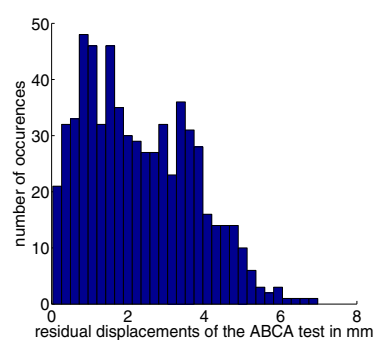

(c)

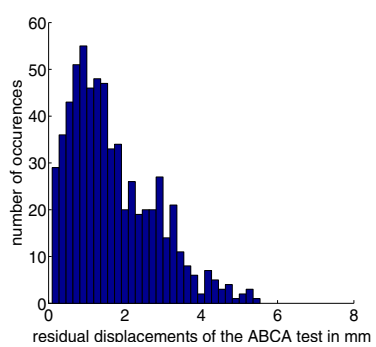

(f)

Fig. 3. $A B C A$ test performed using Methods 1 and 2. a) shows mesh $\mathrm{A}$ after the $A \rightarrow$ $B \rightarrow C \rightarrow A$ registration using Method1. In the ideal case, it should be a regularly tessellated sphere. b) shows displacement vectors between original A and A-registered-backto-itself through B and C. c) shows a histogram of displacement vector norms from b) (mean displacement is approx. $2 \mathrm{~mm}$ ). d), e) and f) show analogous results for Method2. All displacement values are smaller than the length of the mesh triangle side $(\approx 7 \mathrm{~mm})$. Mean (true) displacement values for each of the $A \rightarrow B, B \rightarrow C$ and $C \rightarrow A$ registration steps are $5 \mathrm{~mm}, 6 \mathrm{~mm}$ and $10 \mathrm{~mm}$ respectively for both methods.

and the results are shown in Fig. 3. For every registration step of the $A B C A$ test, the final mean vertex-to-vertex correlations between the input and the reference meshes were approx. 0.98 ( $5 \%$ to $10 \%$ increase from the initial mean vertex-tovertex correlation) indicating successful alignment/convergence for both registration methods. The $A B C A$ test was also performed on thalami from three healthy controls. For Method1, the average vertex displacement from the registrations (A$\mathrm{B}, \mathrm{B}-\mathrm{C}$ and $\mathrm{C}-\mathrm{A}$ ) was approx. $1.5 \mathrm{~mm}$ ( $5 \mathrm{~mm} \max )$. The average residual displacements (A-B-C-A) were approx. $1 \mathrm{~mm}(5 \mathrm{~mm} \max )$ and the average mesh edge length approx. $2 \mathrm{~mm}$. As the ABCA test involves three registrations, the individual registration errors are likely to be less than the total ABCA residual error. For Method2, however, the average vertex displacements from the B-C and C-A registrations were similar to the average residual displacement, while the A-B registration found no displacements. This could be due to convergence problems with the variable metric approach when optimising fine-scale wavelet coefficients, possibly due to the highly non-linear similarity function. Conjugate gradient optimisation method yielded similar results. 


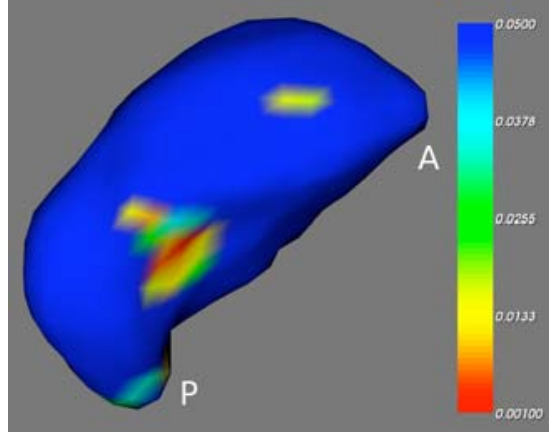

(a)

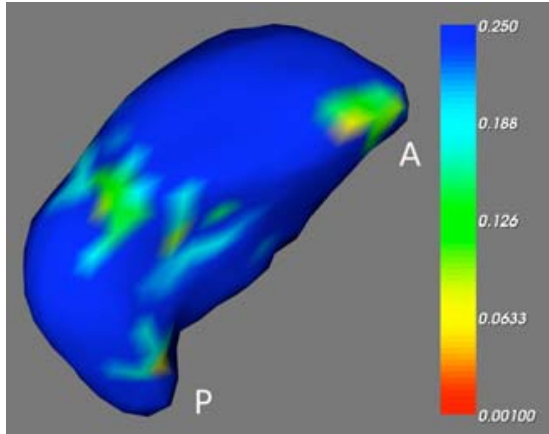

(b)

Fig. 4. Statistical analysis of residual within-surface displacements between control and AD groups for the left thalamus. Colourbars represent uncorrected p-values. a) Results for Method1. b) Results for Method2. A - anterior, $\mathrm{P}$ - posterior.

These methods were further tested on the thalami from a dataset comprising age- and gender-matched AD and control groups (12+12 subjects). An AD subject (the most typical of all 24 subjects [13]) was chosen as template and all others were registered to this using proposed methods. For every vertex, the Euclidean distance between its original position (given by FIRST vertex correspondence) and the new position after registration was compared between AD and control groups. For both methods two-tailed t-tests showed group differences in residual within-surface displacements predominantly in medial dorsal parts of the left thalamus (Fig. 4). Right thalamus showed negligible differences that considerably depended on the choice of the template subject. Spatial positions of changes found in the left thalamus did vary with the choice of template, but were always constrained to the medial dorsal part. Nevertheless, such dependencies demonstrate the potential benefits of construction and investigation of an average template of thalamic connectivity profiles.

\section{Discussion}

We presented a general framework for improving the within-surface correspondence between brain structures based on matching areas of similar structural connectivity patterns. We then introduced two methods implementing this idea. Both perform well on the artificial example suggesting high registration consistency and indicating that the chosen set of features is informative enough to drive the registration. Method2, however, was less sensitive when analysing real data, although it performed better in the artificial examples. We believe this is due to the difficult optimisation step at the highest scale (aligning fine details) where we found the variable metric optimisation approach was often unable to properly converge. This demonstrated the importance of adjusting the optimisation method, as well as the multi-scale approach, to the specific nature of this 
registration problem. Nonetheless, patterns of differences in thalamic connectivity between AD and healthy controls found by both methods are consistent with histological evidence [14. This indicates potential value for the method in determining MRI-based biomarkers of disease progression. Future work will attempt to apply this registration framework to the whole neo-cortical surface.

\section{References}

1. Amunts, K., Schleicher, A., Burgel, U., Mohlberg, H., Uylings, H.B., Zilles, K.: Broca's region revisited: cytoarchitecture and intersubject variability. J. Comp. Neurol. 412(2), 319-341 (1999)

2. Behrens, T., Jenkinson, M., Robson, M., Smith, S., Johansen-Berg, H.: A consistent relationship between local white matter architecture and functional specialisation in medial frontal cortex. NeuroImage 30(1), 220-227 (2006)

3. Johansen-Berg, H., Behrens, T., Sillery, E., Ciccarelli, O., Wheeler-Kingshott, C., Thompson, A., Smith, S., Matthews, P.: Functional-anatomical validation and individual variation of diffusion tractography-based segmentation of the human thalamus. Cerebral Cortex 15, 31-39 (2005)

4. Pascal, C., Jean-Francois, M.: Registration of cortical connectivity matrices. In: CVPRW 2006: Proceedings of the 2006 Conference on Computer Vision and Pattern Recognition Workshop, Washington, DC, USA, p. 66. IEEE Computer Society Press, Los Alamitos (2006)

5. Woolrich, M., Jbabdi, S., Patenaude, B., Chappell, M., Makni, S., Behrens, T., Beckmann, C., Jenkinson, M., Smith, S.: Bayesian analysis of neuroimaging data in FSL. NeuroImage 45, S173-S186 (2009)

6. Behrens, T., Woolrich, M., Jenkinson, M., Johansen-Berg, H., Nunes, R., Clare, S., Matthews, P., Brady, J., Smith, S.: Characterization and propagation of uncertainty in diffusion-weighted MR imaging. Magn. Reson. Med. 50(5), 1077-1088 (2003)

7. Smith, S.: Robust automated brain extraction. In: Sixth Int. Conf. on Functional Mapping of the Human Brain, vol. 625 (2000)

8. Schröder, P., Sweldens, W.: Spherical wavelets: efficiently representing functions on the sphere. In: SIGGRAPH, pp. 161-172 (1995)

9. Schröder, P., Sweldens, W.: Spherical wavelets: Texture processing. In: Hanrahan, P., Purgathofer, W. (eds.) Rendering Techniques, pp. 252-263. Springer, Heidelberg (1995)

10. Yu, P., Grant, P.E., Qi, Y., Han, X., Ségonne, F., Pienaar, R., Busa, E., Pacheco, J., Makris, N., Buckner, R.L., Golland, P., Fischl, B.: Cortical surface shape analysis based on spherical wavelets. IEEE Trans. Med. Imaging 26(4), 582-597 (2007)

11. Nain, D., Haker, S., Bobick, A.F., Tannenbaum, A.: Multiscale 3-d shape representation and segmentation using spherical wavelets. IEEE Trans. Med. Imaging 26(4), 598-618 (2007)

12. Press, W., Teukolsky, S., Vetterling, W., Flannery, B.: Numerical Recipes in C, 2nd edn. Cambridge University Press, Cambridge (1995)

13. Smith, S., Jenkinson, M., Johansen-Berg, H., Rueckert, D., Nichols, T., Mackay, C., Watkins, K., Ciccarelli, O., Cader, M., Matthews, P., Behrens, T.: Tract-based spatial statistics: Voxelwise analysis of multi-subject diffusion data. NeuroImage 31, 1487-1505 (2006)

14. Xuereb, J.H., Perry, R.H., Candy, J.M., Perry, E.K., Marshall, E., Bonham, J.R.: Nerve cell loss in the thalamus in alzheimer's disease and parkinson's disease. Brain 114(pt. 3), 1363-1379 (1991) 